\title{
Single-molecule force spectroscopy quantification of adhesive forces in cucurbit[8]uril host-guest ternary complexes
}

Zarah Walsh-Korb, ${ }^{*, \dagger, \ddagger, \S}$ Ying Yu, ${ }^{\top, \ddagger}$ Emma-Rose Janeček, ${ }^{\dagger}$ Yang Lan, ${ }^{\dagger}$ Jesús del Barrio, $^{\dagger}$ Paul E. Williams, ${ }^{\dagger}$ Xi Zhang, ${ }^{\circledR}$ and Oren A. Scherman ${ }^{*, \dagger}$

$\dagger$ Melville Laboratory for Polymer Synthesis, Department of Chemistry, University of Cambridge, Lensfield Road, Cambridge, CB2 1EW, United Kingdom $\ddagger$ Both authors contributed equally to this work IDepartment of Chemistry, Tsinghua University, Beijing 100084, China §BioTeam, ECPM-ICPEES, UMR 7515, Université de Strasbourg, 25 rue Becquerel, 67087 Strasbourg, Cedex 2, France

E-mail: walshkorb@unistra.fr; oas23@cam.ac.uk

Phone: +33 (0)3 688527 07; +44 (0)1223 331797. Fax: +44 (0)1223336488

\begin{abstract}
Cucurbit[8]uril (CB[8]) heteroternary complexes display certain characteristics making them well-suited for molecular level adhesives. In particular, careful choice of hostguest binding pairs enables specific, fully reversible adhesion. Understanding the effect of the environment is also critical when developing new molecular level adhesives. Here we explore the binding forces involved in the methyl viologen $\cdot \mathrm{CB}[8] \cdot$ naphthol heteroternary complex using single-molecule force spectroscopy (SMFS) under a variety of conditions. From SMFS, the interaction of a single ternary complex was found to
\end{abstract}


be in the region of $140 \mathrm{pN}$. Additionally, a number of surface interactions could be readily differentiated using the SMFS technique allowing for a deeper understanding of the dynamic heteroternary $\mathrm{CB}[8]$ system on the single-molecule scale.

\section{Introduction}

Materials that display reversible or controllable adhesion have gained a large amount of attention in recent years. ${ }^{1-3}$ Such materials are of particular interest in the development of responsive systems, sensors, molecular electronics, biomedicine and biomimetics. ${ }^{2,4,5}$ Nevertheless, their behavior in response to external stimuli is not fully understood and, thus, limits wider application. ${ }^{6,7}$

Recent literature has shown that cucurbit[n]urils $(\mathrm{CB}[\mathrm{n}])$ and cyclodextrins $(\mathrm{CD})$ are applicable in the development of controllable adhesive surfaces on account of their ionic strength, voltage, temperature, $\mathrm{pH}$ and light controlled interactions with a variety of different

guests. ${ }^{1,2,8-10}$ Recent work on the development of responsive surfaces include examples of colloid-colloid, colloid-surface, cell-surface, gel-gel and surface-surface adhesion mediated through $\mathrm{CB}[\mathrm{n}]$ or $\mathrm{CD}$ interactions. ${ }^{9,11-20}$

Important factors in the development of such materials are the adhesive forces of each complex on the interacting surfaces, how individual interactions contribute to bulk adhesion and how they behave relative to their environment over time. Despite these important factors, relatively few reports discuss the interactions on the single molecule level. Yet, it is these single molecule interactions that largely affect the bulk behavior of such adhesive materials. ${ }^{2,3}$ Within this developing field of supramolecular adhesive materials, the cucurbit[n]uril family of macrocycles, in particular cucurbit[8]uril (CB[8]), show significant promise. The ability of $\mathrm{CB}[8]$ to simultaneously encapsulate two guests in its cavity gives this macrocyclic host an unprecedented level of responsiveness to a variety of external stimuli. This responsive behavior also provides the complex with a high level of reversibility making it an appropriate choice for materials requiring repetitive adhesive interactions. While the adhesive behavior 
at the single-molecule level has previously been discussed for $\mathrm{CB}[6], \mathrm{CB}[7]$ and $\beta$-CD, as yet only the bulk adhesive properties of $\mathrm{CB}[8]$ complexes have been examined in recent literature. ${ }^{3,21-23}$ For example, the adhesion of single colloids or nanoparticles to surfaces through simple CB[8] ternary complexes or catenated structures has been reported. ${ }^{24}$ Interactions at surfaces can also be controlled by photochemical stimuli, for example the modulation of surface adhesion through the isomerization of azobenzene derivatives, however, until now no single molecule adhesion behavior has been reported. ${ }^{11,25}$

Unlike binary $\mathrm{CB}[\mathrm{n}]$ host-guest systems exploiting smaller $\mathrm{CB}$ homologs, the dynamic nature of $\mathrm{CB}[8]$ heteroternary complexes coupled with the conformational switching behavior of azobenzenes make such photo-switchable systems inherently difficult to study at the single-molecule level. Therefore, as an initial study the methyl viologen $\cdot \mathrm{CB}[8] \cdot$ naphthol system, which, although reversible, does not undergo conformation changes, will be examined in order to simplify the output from single-molecular experiments. Basic understanding of heteroternary complexation from this initial study will aid the development of more complex CB[8]-based adhesive systems and allow for straightforward characterization and quantification.

Atomic force microscopy (AFM)-based single-molecule force spectroscopy (SMFS) allows for the precise measurement of minute forces at a single-molecule level. This technique not only allows interactions to be directly measured ${ }^{26}$ but also provides new physical insight into the interactions within polymer chains ${ }^{27}$ or dsDNA. ${ }^{28}$ Here we explore the adhesive properties of $\mathrm{CB}[8]$ ternary complexes and quantify them at the single-molecule scale by SMFS. Figure 1, shows a pictoral overview of the experimental system.

Poly(dimethylacrylamide) (PDMA) chains of varying lengths were synthesized by reversible addition-fragmentation chain transfer (RAFT) polymerization and used to separate a gold-coated AFM tip from the naphthol second guest in order to eliminate non-specific interactions. Detailed studies on the effect of polymer chain length, macrocyclic host molecule $(\mathrm{CB}[8]$ or $\mathrm{CB}[7]$ as a control) and the electrochemical potential led to the elucidation of 


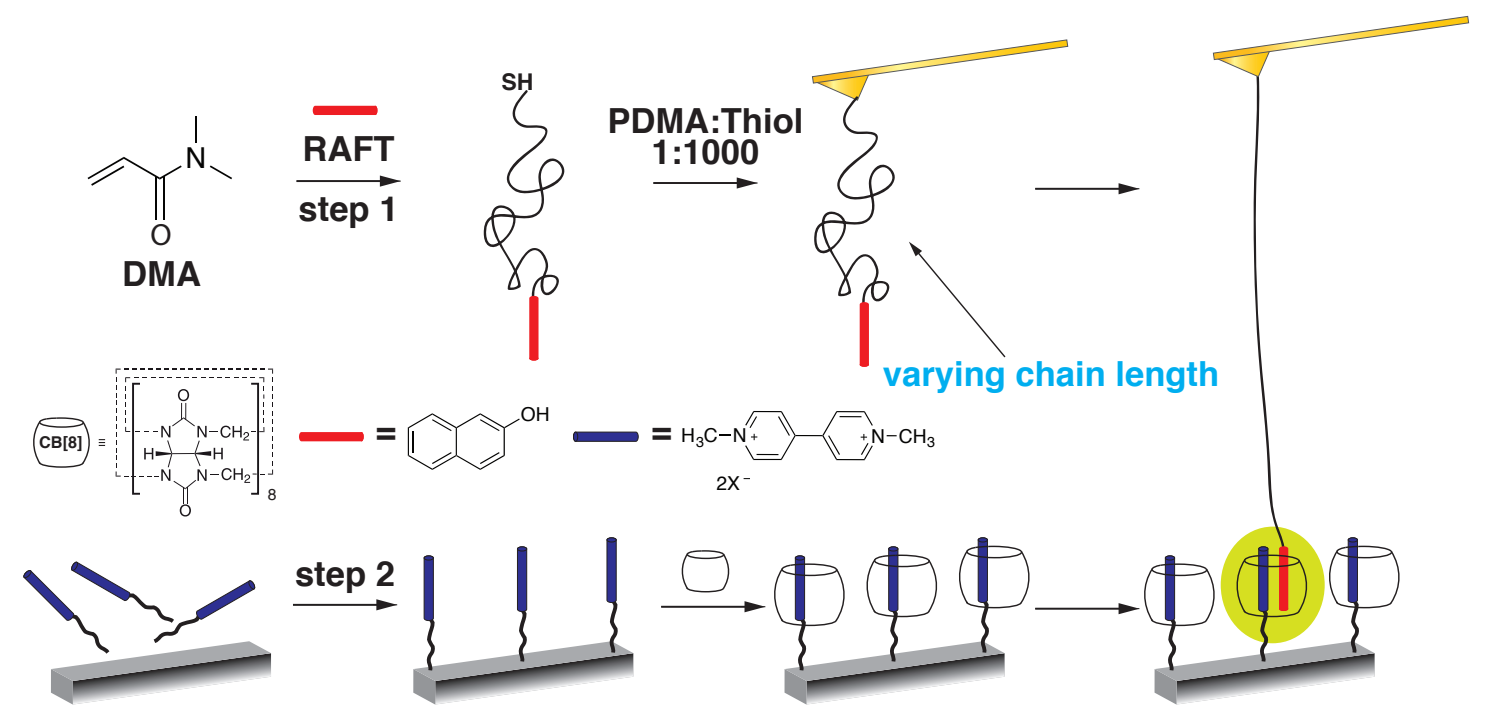

Figure 1: Schematic representation of the functionalization of the surface and AFM tip and subsequent pulling experiments.

three different interactions at the surface enabling quantification of their relative interaction forces, which are discussed in detail in the following sections. The binding force of the heteroternary complex in CB[8] was found to be approx. $140-160 \mathrm{pN}$, a value that is in good agreement with other $\mathrm{CB}[\mathrm{n}]$ binding forces quantified by force spectroscopy reported in the literature, for example, the CB[6]-spermine binary complex (approx. $120 \mathrm{pN}$ ). ${ }^{23}$

\section{Experimental Procedures}

Single-molecule force spectroscopy (SMFS) experiments All single-molecule force spectroscopy experiments were carried out on an MFP3D atomic force microscope (Asylum Research, Santa Barbara, CA, USA) using TR400PB probes (Olympus, Tokyo, Japan), which are coated on both sides with a thin gold layer. Probes were either used untreated or functionalized according to the procedure described in the following section. All SMFS experiments were carried out on freshly cleaved and UV-plasma treated mica (Elektron Technologies, Stansted, UK) or surfaces that had been functionalized with a methyl viologen silane (see below) and stored in air-tight containers before use. Experiments to determine the 
force of interaction of second guests in the CB[8] ternary complex on the mica surface were carried out in an aqueous solution of $0.1 \mathrm{mM}$ cucurbit[8]uril (CB[8]). Control experiments were carried out in solutions of either $0.1 \mathrm{mM}$ cucurbit[7]uril $(\mathrm{CB}[7])$ or purified water. Reduction experiments were carried out in a $33 \mathrm{mM}$ solution of sodium dithionite $\mathrm{Na}_{2} \mathrm{~S}_{2} \mathrm{O}_{4}$ purchased from Sigma Aldrich Chemical (Shanghai, China).

Preparation and functionalization of poly(dimethylacrylamide) Firstly, a hydroxylated chain transfer agent $(\mathrm{CTA}-\mathrm{OH})$ was prepared according to a procedure described by Biedermann et al.. ${ }^{29}$ Subsequently, CTA-OH (130 mg, $\left.0.5 \mathrm{mmol}\right)$, N,N'-dimethyl acrylamide $(1.5 \mathrm{~g}, 1.56 \mathrm{~mL}, 15 \mathrm{mmol})$ and ACPA (13 mg, $0.05 \mathrm{mmol})$ were dissolved in 1,4-dioxane $(7 \mathrm{~mL})$. The solution was bubbled with nitrogen for $1 \mathrm{~h}$, then heated to $70{ }^{\circ} \mathrm{C}$ for $6 \mathrm{~h}$. The solution was then quenched in liquid $\mathrm{N}_{2}$ and precipitated into cold diethyl ether. The diethyl ether was decanted off and the residual polymer dissolved in THF. The polymer was isolated under reduced pressure as a yellow solid (0.92 g). The degree of polymerization (DP) was determined by NMR end group analysis to be 8.9. The number average molecular weight $\left(\mathrm{M}_{n}\right)$, the weight average molecular weight $\left(\mathrm{M}_{w}\right)$ and the polydispersity index (PDI) were determined to be $2500 \mathrm{~g} / \mathrm{mol}, 3000 \mathrm{~g} / \mathrm{mol}$ and 1.2, respectively, as determined by GPC with THF as eluent. When targeting other DPs the above procedure was followed with the exception that higher ratios of CTA-OH and ACPA were used, resulting in increased $\mathrm{M}_{n}$ and $\mathrm{M}_{w}$ as shown in Table 1. The quantities of the CTA-OH and ACPA along with the resultant $\mathrm{M}_{n}, \mathrm{M}_{w}$ and PDI are shown in Table 1.

Polymers were then denoted $\mathrm{DMA}_{x}$ where $x=5 k, 12 k$ or $19 k$, roughly the $\mathrm{M}_{n}$ values for each of the synthesized polymers.

End-group functionalization $\mathrm{DMA}_{x}$ and naphthol-isocyanate were dissolved, in the concentrations shown in Table 2, in DCM (5-10 mL) followed by the addition of 1 drop of dibutyltindilaurate.

The mixture was stirred overnight. The solvent was then partially evaporated under 
Table 1: Quantities of CTA-OH and ACPA used to synthesize longer spacer chains along with the resultant $\mathrm{M}_{n}, \mathrm{M}_{w}$ and PDI

\begin{tabular}{l|c|c|c|c|c} 
Sample & CTA-OH, mmol & ACPA, mmol & $\mathbf{M}_{n}, \mathbf{g} / \mathbf{m o l}$ & $\mathbf{M}_{w}, \mathbf{g} / \mathbf{m o l}$ & PDI \\
\hline DMA $_{5 k}$ & $0.23(60 \mathrm{mg})$ & $0.02(6 \mathrm{mg})$ & 4900 & 6300 & 1.3 \\
DMA $_{12 k}$ & $0.076(19.6 \mathrm{mg})$ & $0.007(2 \mathrm{mg})$ & 11900 & 13900 & 1.2 \\
DMA $_{19 k}$ & $0.0151(4 \mathrm{mg})$ & $0.0014(0.4 \mathrm{mg})$ & 19500 & 23800 & 1.2
\end{tabular}

Table 2: Concentrations and ratios of $\mathrm{DMA}_{x}$ and naphthol-isocyanate (Np-NCO) used for end-group functionalization

\begin{tabular}{l|c|c|c|c} 
Sample & $\mathbf{D M A}_{x}, \mathbf{m g}$ & $\mathbf{D M A}_{x}, \mathbf{m m o l}$ & $\mathbf{N p}-\mathbf{N C O}, \mathbf{m g}$ & $\mathbf{N p}-\mathbf{N C O}, \mathbf{m m o l}$ \\
\hline $\mathbf{D M A}_{5 k}$ & 200 & 0.04 & 25 & 0.147 \\
$\mathbf{D M A}_{12 k}$ & 200 & 0.017 & 10 & 0.059 \\
DMA $_{19 k}$ & 200 & 0.01 & 6 & 0.035
\end{tabular}

reduced pressure and the crude mixture precipitated from cold diethyl-ether. The isolated polymer was then re-dissolved, filtered and re-precipitated. These functionalized polymers were then denoted Np-DMA $x$. The unfunctionalized DMA $\mathrm{D}_{x}$ consists of a methoxyphenyl end group as a result of the CTA-OH unit. As this unit has a $\mathrm{K}_{a}$ value with $\mathrm{MV}^{2+} \cdot \mathrm{CB}[8]$ at least two orders of magnitude lower than that of the naphthol unit, ${ }^{30}$ this chain was used as the negative control for the procedure and denoted Pm-DMA ${ }_{x}$.

Isothiocyanate cleavage Polymer was dissolved in $4.5 \mathrm{~mL}$ of methanol such that the concentration of polymer was between 0.6 and $1 \mu \mathrm{mol}$ (4-25 mg depending on the $\mathrm{M}_{n}$ of the polymer). To this solution was added $0.5 \mathrm{~mL}$ of concentrated $\mathrm{HCl}$ and the mixture was heated to reflux under inert atmosphere for $4-5 \mathrm{~h}$. The resulting cleavage solution was 
refrigerated until required for tip functionalization.

Functionalization of AFM tips Use of the cleaved polymer directly after cleavage resulted in a significantly higher number of polymer chains attached to the tip than the required single unit. ${ }^{31}$ A $33 \mathrm{mM}$ solution of mercaptohexanol in ethanol was then prepared. Prior to functionalization the AFM tips were washed in ethanol and then immersed in the mercaptohexanol solution for 20 min. After 20 min the cleaved polymer solution was added in a 1:1000 ratio with the mercaptohexanol solution. The volume of addition was varied to keep the molar ratio consistent between all functional polymers. The functional polymer was allowed to remain in solution with the AFM tip for 10 min before the AFM tips were removed and washed thoroughly with ethanol and then water. The tips were then generally used immediately following functionalization up to a maximum of 5 days post-functionalization in order to maintain the reproducibility of our results by limiting tip contamination.

Functionalization of the mica surfaces Mica surfaces were functionalized with a methyl viologen silane prepared according to the procedure previously described by this group, with the exception that the methyl viologen contained only one silane functionality per molecule. ${ }^{32}$ The surface counterion was changed by immersing the surfaces in a concentrated solution of ammonium tetrafluoroborate or sodium chloride overnight as required. Functionalized surfaces were characterized prior to SMFS experiments using contact angle measurements (see ESI, Figure S1) to determine if the surface functionalization had been successful. This was carried out on an FTA22000 optical contact angle instrument (First Ten Ångstroms, Portsmouth, VA, USA) using dynamic contact angle measurements.

Verification of interaction of ternary complex To validate association/dissociation constant data of the ternary complexes obtained by SMFS, solution-based studies were also carried out using isothermal titration calorimetry (ITC) on a Nano-ITC system from TA Instruments (Elstree, UK) with a $1 \mathrm{~mL}$ cell volume and deionized water from a Milli-Q 
water purification system as the reference liquid. Due to solubility issues of the Np-DMA in water at the concentrations required for ITC, the Np-DMA $x$ was replaced for this analysis with $\mathrm{Np}-\mathrm{PEG}_{5 k}$, synthesized from a procedure described by Rauwaldet al. ${ }^{33}$

\section{Results and Discussion}

In order to understand the interaction behavior of a $\mathrm{CB}[8]$ ternary complex at the single molecule scale, and its effect on adhesion, a well-understood system was chosen. Therefore, the system could be better controlled with the resulting behavior more easily understood. The system studied consisted of methyl viologen $\left(\mathrm{MV}^{2+} 2\left(\mathrm{X}^{-}\right), 1^{\text {st }}\right.$ guest), 2-naphtholterminated PDMA $\left(\mathrm{Np}-\mathrm{DMA}_{x}, 2^{\text {nd }}\right.$ guest), and $\mathrm{CB}[8]$, shown in Figure 1, and has been described in the literature many times. The polymeric linker is not expected to play a role in the specific interactions of the system; its role is to ensure a significant distance between the napthol unit, and thereby the ternary complex, from the AFM tip to ensure no tip-surface interactions affect the behavior of the ternary complex.

The effect of the polymer linker in the system Several preliminary SMFS experiments were carried out with a naphthol silane directly attached to the AFM tip, however the resulting spectra were so complicated to analyse it was clear that ternary complex formation was not the only process observed in the experiment. It is likely that a variety of tip-surface interactions were being probed simultaneously and the interaction of the gold-coated tip with the highly charged $\mathrm{MV}^{2+}$ surface and the portals of the $\mathrm{CB}[8]$ were dominating the spectra making interpretation of any ternary complex interaction forces impossible. It was decided that a polymer linker should be introduced into the system to separate the tip significantly from the ternary complex so that no tip-surface interactions would be visible in the resulting SMFS data. It was unclear how long the required linker should be in order to sufficiently separate the tip from the rest of the system, thus three PDMA polymers of $\mathrm{DMA}_{5 k}, \mathrm{DMA}_{12 k}$ and $\mathrm{DMA}_{19 k}$ were synthesized and functionalized with a 2-naphthol moiety. 
In order to understand the effect of the linker in the system a series of cantilevers were functionalized, using the procedure described in the experimental section, with each of the three different Np-terminated PDMA linkers. Using the standard $\mathrm{MV}^{2+} 2\left(\mathrm{BF}_{4}{ }^{-}\right)$surfaces a series of SMFS experiments were performed under the same conditions of approach speed $(500 \mathrm{~nm} / \mathrm{s})$ and dwell time $(1 \mathrm{~s})$ in water both in the presence and absence of CB[8]. Representative histograms showing the frequency of the different interaction forces for each of the three Np-DMA polymers are shown in Figure 2. Histograms showing the distribution of interaction lengths for Np-DMA5k, Np-DMA12k and Np-DMA19k, as well as control experiments for the system are shown in the ESI (Figure S2 and S5). Interaction forces are calculated by subtracting the baseline of the force spectra from the average height of the plateau or the apex of a peak or plateau-peak, examples of raw data are shown in Figure S4, as well as Figure 4.
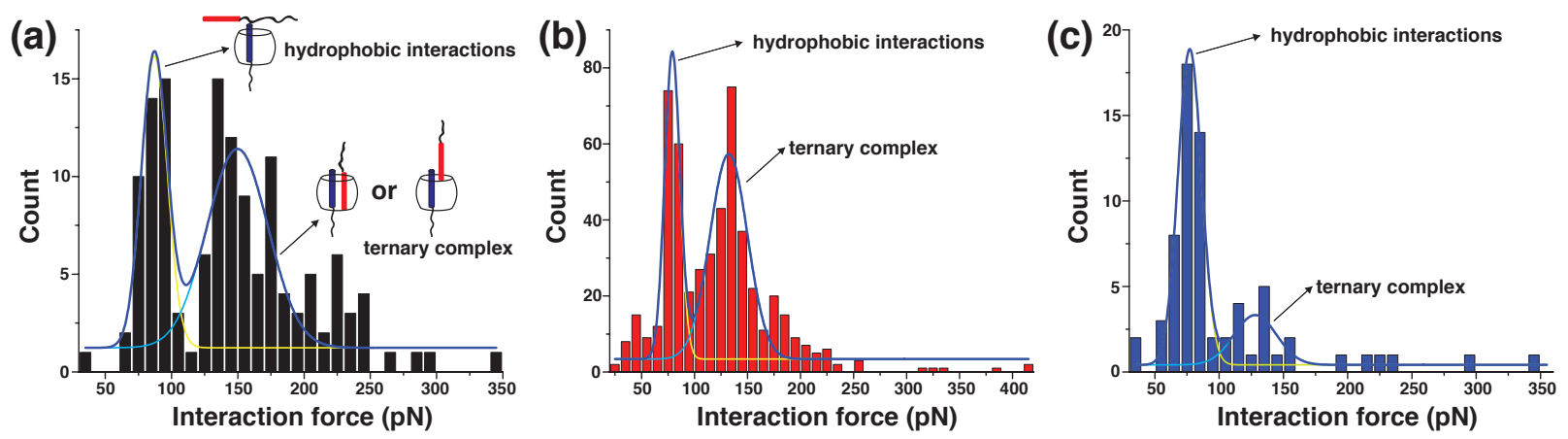

Figure 2: Histograms showing the distribution of interaction forces for (a) $\mathrm{Np}^{-\mathrm{DMA}_{5 k}}$, (b) Np-DMA $12 k$ and (c) Np-DMA $19 k$

Looking first at Figure 2a and the corresponding length histogram, Figure S5a, the data for the interactions between the $\mathrm{MV}^{2+} 2\left(\mathrm{BF}_{4}^{-}\right) \cdot \mathrm{CB}[8]$ surface with the Np-DMA $5 k$ polymer functionalized AFM tip are shown. In SMFS, a specific binding event is defined as any peaks observed in the spectrum well resolved from the initial surface contact peak, that is, the non-specific binding interaction. From the individual spectra (example shown in ESI, Figure S4a), it is clear that it is difficult to differentiate the binding event (secondary peaks and plateau, which are discussed in more detail in a later section) from the non- 
specific interactions (large peak), this is likely due to the short length of the polymer. As the polymer is short and the non-specific interactions are large, the non-specific interactions dominate the spectra and can skew the determined interaction forces and length. This effect can be seen in both Figure 2a and Figure S5a. A clear bimodal distribution exists centered at approximately $80 \mathrm{pN}$ but the stronger interactions have a wide distribution up to $250 \mathrm{pN}$ with no clear center. The bimodal distribution indicates the relative frequency of nonspecific hydrophobic interactions (peak centered below $100 \mathrm{pN}$ ) to specific ternary complex or partial ternary complex interactions (peak centered above $100 \mathrm{pN}$ ), the data was fitted using a Gaussian peak fitting model. Figure S5a shows that the interaction length is long and disperse, ranging from 150 to $450 \mathrm{~nm}$, giving no clear indication of the apparent polymer length. Comparing the information from the histograms with the representative spectra, it can clearly be seen in the case of the $\mathrm{Np}-\mathrm{DMA}_{5 k}$ polymer that the non-specific interactions dominate the spectra. From a total of 744 recorded interactions in 1024 experiments, 290 appear to be specific interactions at the portal of the CB[8] (28\%). It is thus likely that the $5 \mathrm{kDa}$ polymer chain is too short to fully separate bulk tip-surface interactions from the interactions of the single molecules on the AFM tip and the surface forming host-guest complexes, and the result is a complicated data set from which accurate data is difficult to obtain.

Increasing the polymer length will increase the interaction time the naphthol moiety has with the $\mathrm{CB}[8]$ on the surface during retraction, which allows for easier differentiation between specific and non-specific interactions. Examining the individual force spectra (ESI, Figure S4b), the various interaction events are well separated from the non-specific interaction peak. Effective separation of the specific and non-specific events allows more accurate characterization of the single molecule interactions. Figure $2 \mathrm{~b}$ shows the distribution of interaction forces obtained from a polymer linker of $\mathrm{M}_{n}=12 \mathrm{k}, \mathrm{Np}-\mathrm{DMA}_{12 k}$. Again there appears to be a bimodal distribution of interaction forces with one peak centered at approximately $80 \mathrm{pN}$ and a second at approximately $140 \mathrm{pN}$. With the increased chain length, $48 \%$ of 
recorded interactions (515 in 1024) appear to be specific interactions at the $\mathrm{CB}[8]$ portal. Here the distribution is far more clearly seen due to the increased frequency of events with this longer polymer chain. It is also worth noting that, despite the clarity of the spectra, the specific interaction peak is more disperse than that of the non-specific interaction peak. As the hydrophobic interactions are static, one can expect the recorded pull-off force to be almost the same on each occasion, hence the narrow distribution of recorded forces. In the case of specific and partial ternary complex formation, the recorded forces cover a wider range. These differences are likely due to the dynamic nature of the ternary complex. It has been reported that the $\mathrm{CB}[8]$ ternary complex is not static but that the $\mathrm{CB}[8]$ shuttles back and forth over the two guest units inside the cavity. ${ }^{34}$ This would mean that the force required to remove the second guest at any given moment may be different depending on the location of the host unit with respect to the guests, resulting in a larger dispersion of ternary complex interaction forces, compared to interaction forces observed for static, nonspecific interactions. The increased polymer chain length increases the distance between the functional group and the AFM tip. Additionally, within the timescale of the force measurement, the naphthol moiety spends more time near the $\mathrm{CB}[8]$ functionalized surface resulting in an increased likelihood of a specific interaction event. As the specific and non-specific interactions of the system are well separated from one another the interaction length is more clearly defined, centered at approximately $100 \mathrm{~nm}$ (Figure S5b). The data suggests that the polymer extends approximately $100 \mathrm{~nm}$ before the interaction with the surface is terminated and the tip moves to another location. A value of $100 \mathrm{~nm}$ correlates is quite a lot longer than the calculated contour length of PDMA with a DP of 120, which should be approx. $30 \mathrm{~nm}$. Further study is required to determine the reliability of polymer length characterization for this system using SMFS.

Increasing the polymer linker length further to an $\mathrm{M}_{n}$ value of $19 \mathrm{kDa}, \mathrm{Np}-\mathrm{DMA}_{19 k}$, does not yield better resolution between specific and non-specific peaks. In fact, multiple peaks and hydrophobic interactions occur simultaneously or in such quick succession as to 
make interpretation of the spectra more complicated. This can be seen in the representative spectrum shown in Figure S4c. Estimation of the number of different peak types was complicated and it is unclear if the resulting frequency count is an accurate representation of the surface interactions as only 68 events were recorded in 1024 attempts, of which only $15(1.5 \%)$ appear to be interactions at the portal (Figure 2c). Likely the polymeric linker is now so long that it is capable of forming multiple interactions during tip retraction. It is important for the analysis of the data that the tip and linker are completely retracted from the surface once during each pull-off event otherwise the output of the force of interaction can be misinterpreted. While the characteristic bimodal distribution is again present (Figure 2c), the number of occurrences is relatively low (22\% of 68 events) compared to that (48\%) for the Np-DMA $12 k$ polymer. Here, the interaction length is centered at approximately $60 \mathrm{~nm}$ (Figure S5c) much closer to the calculated value of $50 \mathrm{~nm}$ for PDMA with DP of 196, however the number of occurrences are too low to be reliable. A shorter interaction length for a longer polymer chain suggests that either the polymer is not fully removed from the surface during retraction or that the polymer is not completely extended during interaction with the surface, but rather entangled or wrapped around the cantilever. Any of these scenarios presents complications in the interpretation of the data (clearly seen in Figure 2c) and, therefore, it was determined that $\mathrm{Np}-\mathrm{DMA}_{19 k}$ as well as $\mathrm{Np}-\mathrm{DMA}_{5 k}$ were unsuitable for the progression of this study.

It has been previously reported that a theoretical 'sweet spot' exists for linker lengths in SMFS experiments depending on the force of interaction at the surface, the surface coverage, and the dwell time. This is primarily in relation to the use of poly(ethylene glycol) (PEG) linkers, most commonly used in SMFS experiments. While PEG chains of 25-50 nm are most commonly used, ${ }^{35-39}$ it has also been shown that using shorter PEG chains of approximately $800 \mathrm{Da}(8-10 \mathrm{~nm})$ can significantly improve resolution of peaks in SMFS. ${ }^{40}$ Calculated contour lengths of the three different Np-DMA $\mathrm{A}_{x}$ polymers were $12 \mathrm{~nm}, 30 \mathrm{~nm}$ and $50 \mathrm{~nm}$, respectively. With its suitable length, the $\mathrm{DMA}_{12 k}$ linker was chosen for all 
further experiments. The fact that a medium linker $(30 \mathrm{~nm})$, rather than a short one, is required to fully separate the tip and surface when using PDMA relative to PEG may be due to the increased hydrophobicity of the PDMA, as chains may not fully extend into the aqueous medium into which they are immersed, thus polymers with a longer contour length are required to achieve the level of resolution that may be achieved with a shorter PEG linker.

Understanding the effect of the counterion in the solution state Many publications report various phenomena related to the use of different counterions on the $\mathrm{MV}^{2+}$ first guest, several of these refer to the fact that the specific counterion under investigation aids or hinders the formation of the ternary complex. Preliminary experiments suggested that adhesion is enhanced in the presence of a $\mathrm{Cl}^{-}$counterion compared to $\mathrm{BF}_{4}{ }^{-}$. This observation has been inferred previously in the literature but not studied in detail at the single-molecule level.

Methyl viologen with $\mathrm{BF}_{4}^{-}, \mathrm{Cl}^{-}$, and $\mathrm{Br}^{-}$counterions was prepared and isothermal titration calorimetry (ITC) was used to determine if these different counterions would have an effect on the association, $\mathrm{K}_{a}$, and dissociation, $\mathrm{K}_{d}$, constants for the $\mathrm{MV}^{2+} 2\left(\mathrm{X}^{-}\right) \cdot \mathrm{CB}[8] \cdot \mathrm{Np}$ system. While bulk or solution behavior is not necessarily representative of the behavior of a single molecule system, due to competitive and complementary interactions, it provides a useful starting point in understanding environmental effects on the formation of the ternary complex.

For solution based tests $\mathrm{Np}-\mathrm{DMA}_{x}$ was replaced with $\mathrm{Np}-\mathrm{PEG}_{5 k}$ on account of solubility issues in water. This change of polymer system was deemed to be insignificant as the $\mathrm{PEG}_{5 k}$ simply acts to increase solubility of the Np moiety in water and the DMA only serves to remove tip-surface interactions, therefore, neither polymer plays a role in specific binding. Using this modified system, the $\mathrm{K}_{a}$ and $\mathrm{K}_{d}$ values of the binding of the Np unit into the $\mathrm{MV}^{2+} \cdot \mathrm{CB}[8]$ binary complex with the three different counterions were obtained and the data are presented in Table 3 . 
Table 3: Effect of $\mathrm{MV}^{2+}$ counterions on $\mathrm{K}_{a}$ and $\mathrm{K}_{d}$ measured by ITC

\begin{tabular}{l|c|c} 
Complex & $\mathbf{K}_{a}\left(\mathbf{M}^{-1}\right)$ & $\mathbf{K}_{d}(\mathbf{M})$ \\
\hline $\mathrm{MV}^{2+} 2\left(\mathrm{BF}_{4}^{-}\right) \cdot \mathrm{CB}[8] \cdot \mathrm{Np}-\mathrm{PEG}_{5 k}$ & $5.420 \times 10^{4}$ & $1.845 \times 10^{-5}$ \\
$\mathrm{MV}^{2+} 2\left(\mathrm{Cl}^{-}\right) \cdot \mathrm{CB}[8] \cdot \mathrm{Np}-\mathrm{PEG}_{5 k}$ & $6.802 \times 10^{4}$ & $1.470 \times 10^{-5}$ \\
$\mathrm{MV}^{2+} 2\left(\mathrm{Br}^{-}\right) \cdot \mathrm{CB}[8] \cdot \mathrm{Np}-\mathrm{PEG}_{5 k}$ & $5.629 \times 10^{4}$ & $1.776 \times 10^{-5}$
\end{tabular}

As can be seen in Table 3, no significant difference exists between the $\mathrm{K}_{a}$ and $\mathrm{K}_{d}$ values for each of the three systems, suggesting there is no macroscopic difference between them in solution. The enhanced adhesive effect is therefore likely to be a result of the reduced dimensions of the environment and the effect on single interactions.

Varying the counterion on the surface To probe this effect in more detail the $\mathrm{BF}_{4}{ }^{-}$and $\mathrm{Cl}^{-}$systems were chosen for further study. Experiments were carried out with Np-DMA $12 k$ functionalized tips as used in the previous section in the presence of water to determine the baseline interactions of both systems (ESI Figure S3). Detailed pull-off experiments in the presence of $\mathrm{CB}[8]$ were repeated at a pulling speed of $500 \mathrm{~nm} / \mathrm{s}$ and a dwell time of $1 \mathrm{~s}$ for a total of 1024 sampling locations approximately $50 \mathrm{~nm}$ from each other. A histogram of interaction force distributions of the $\mathrm{MV}^{2+} 2\left(\mathrm{Cl}^{-}\right)$system can be seen in Figure 3 .

The information in Figure $2 \mathrm{~b}$ and Figure $\mathrm{S} 7$ shows the data obtained from the $\mathrm{BF}_{4}{ }^{-}$ surface for comparison with the $\mathrm{Cl}^{-}$surfaces. The interaction of $\mathrm{MV}^{2+} 2\left(\mathrm{BF}_{4}{ }^{-}\right) \cdot \mathrm{CB}[8]$ with the $\mathrm{Np}-\mathrm{DMA}_{12 k}$ shows two distinct interaction forces at approximately $80 \mathrm{pN}$ and $140 \mathrm{pN}$ and an interaction length of approximately $90 \mathrm{~nm}$. Comparing this with the data obtained for the $\mathrm{Cl}^{-}$surface (Figure 3 and Figure S6 shows the same pattern of interactions but the values of these interactions are increased to approximately $90 \mathrm{pN}$ and $160 \mathrm{pN}$, although the interaction length remains roughly the same. The non-specific interaction peaks in both systems (ESI, Figure S7a,b) shows that on average the non-specific interaction in the $\mathrm{BF}_{4}{ }^{-}$ 


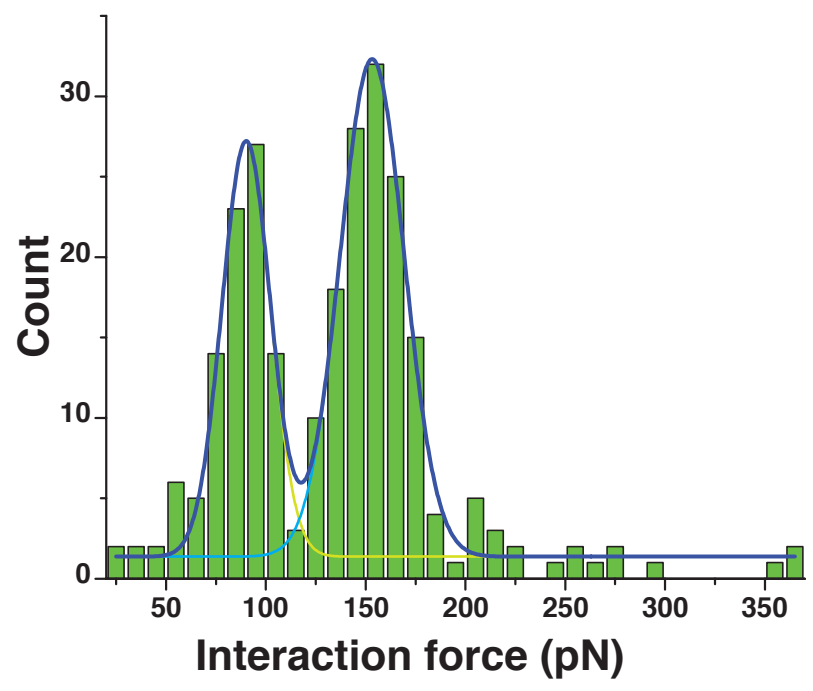

Figure 3: Histogram showing the distribution of interaction forces for $\mathrm{MV}^{2+} 2\left(\mathrm{Cl}^{-}\right) \cdot \mathrm{CB}[8] \cdot \mathrm{Np}-\mathrm{DMA}_{12 k}$

system is on the order of $1 \mathrm{nN}$, while in the $\mathrm{Cl}^{-}$system that value increases to approximately $1.5 \mathrm{nN}$.

From previous work we have estimated the MV surface density to be $50-300 \mathrm{~nm}^{2}$, this variation in coverage depends on the counterions used in the functionalization procedure. ${ }^{32,41}$ In general larger counterions result in lower surface density such that the $\mathrm{BF}_{4}{ }^{-}$system has a spacing of $300 \mathrm{~nm}$ while the $\mathrm{Cl}^{-}$are only $50 \mathrm{~nm}$ apart. Taken together the increased values of both the specific and non-specific interactions, coupled with the higher surface density of $\mathrm{MV}^{2+}$ moieties with $\mathrm{Cl}^{-}$counterions explains the increased adhesive properties of the $\mathrm{Cl}^{-}$ system.

Understanding the various peak formations (manipulation of the surface) Throughout this work, all of the interaction force histograms have displayed distinctly bimodal distributions. Looking more closely at the histograms and the respective spectra, three different spectral patterns emerged. The lower interaction force region is the easiest to interpret as only one spectral pattern coincides with this interaction force. This specific pattern can be seen in Figure 4b and will be referred to as a plateau.

The plateau likely corresponds to hydrophobic interactions between the functionalized 

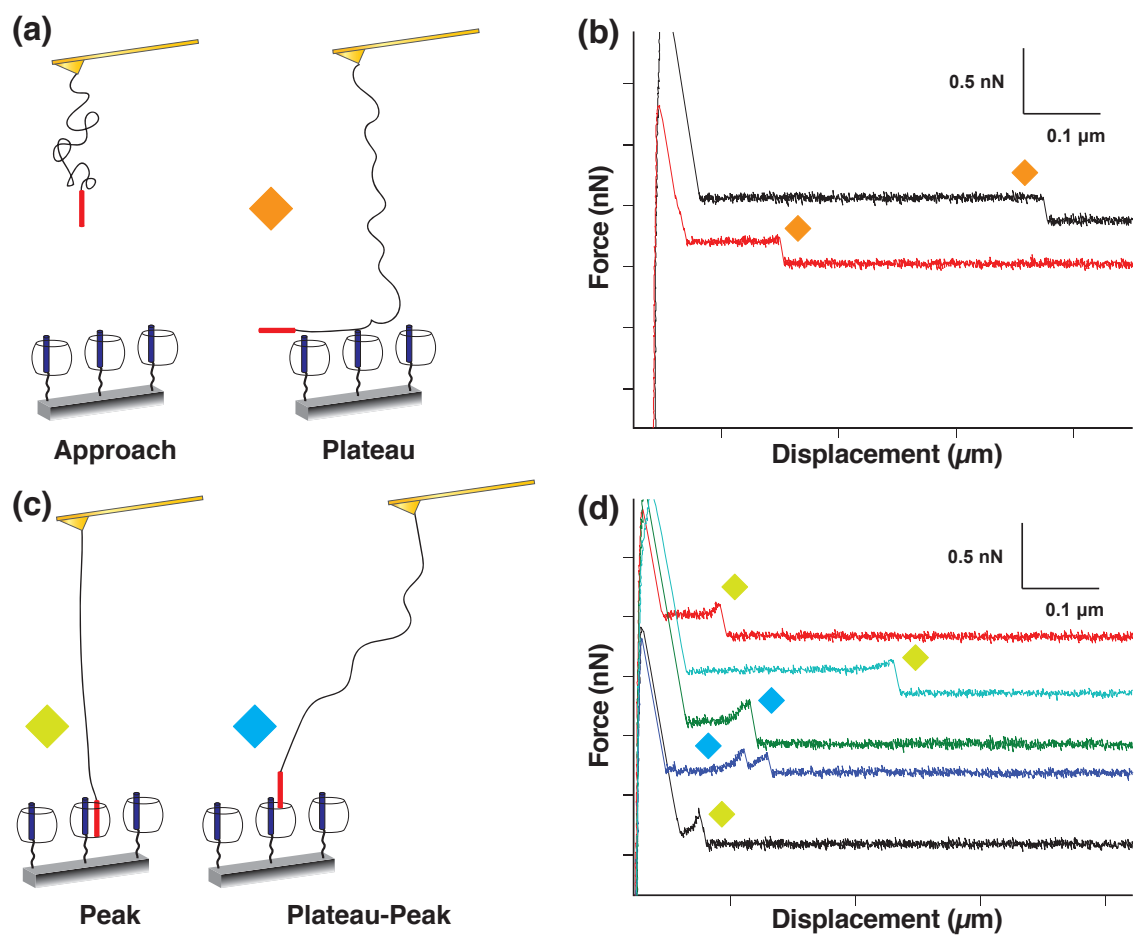

Figure 4: Representative SMFS spectra of the $\mathrm{MV}^{2+} 2\left(\mathrm{BF}_{4}^{-}\right) \cdot \mathrm{CB}[8] \cdot \mathrm{Np}-\mathrm{DMA}_{12 k}$ experiments showing (a) a graphical representation of the approach and the orientation of complexing units resulting in plateau in the spectral data (b) examples of plateau observed in the SMFS experiments (c) a graphical representation of the the orientation of complexing units resulting in peaks and plateau-peaks in the spectral data and (d) examples of peaks and plateau-peaks observed in the SMFS experiments

polymer chain and the surface, and is a result of the polymer chains 'lying' on the functional surface as depicted in Figure 4a. Due to the weak interaction a strong pull is not required from the AFM tip to terminate it, and therefore no change occurs in the interaction force until pull-off. The stronger interaction forces were more difficult to determine as they produced two different interaction patterns within roughly the same pull-off force. These two different interaction patterns are referred to as peaks and plateau-peaks and are shown in Figure 4c and $4 \mathrm{~d}$, a pictoral representation and the spectral pattern, respectively. The peak pattern, as would be expected, is characterized by a sharp increase at the end of a plateau with an interaction force in the region of $140 \mathrm{pN}$. This is force of the ternary complex interaction between the naphthol on the AFM tip and the $\mathrm{MV}^{2+} \cdot \mathrm{CB}[8]$ complex on the mica surface. The plateau-peak on the other hand is characterized by a curved, often flattened peak at 
the end of the plateau indicated with blue diamonds in Figure 4c. This plateau-peak has an interaction force in the region of $130 \mathrm{pN}$ but was overall a rather rare occurrence in the course of these experiments, less than $10 \%$ of all observed interactions; nevertheless, it was important to find its source. It was hypothesized that the plateau-peak was related to incomplete complexation of the naphthol remaining at the portal of the $\mathrm{CB}[8]$.

To test this hypothesis, sodium thiosulfate $\mathrm{Na}_{2} \mathrm{~S}_{2} \mathrm{O}_{4}$ was added to $\mathrm{CB}[8]$ surrounding the tip and surface, in order to reduce the $\mathrm{MV}^{2+}$ to its radical cation $\left(\mathrm{MV}^{+\cdot}\right)$, eliminating ternary complexation of the naphthol. Upon addition of the $\mathrm{Na}_{2} \mathrm{~S}_{2} \mathrm{O}_{4}$ the surface was analyzed and the occurrence of the plateau-peak greatly increased, in fact only plateau and plateau-peaks were observed with the viologen in its radical cation state. As the liquid cell of the AFM is not closed, oxygen is able to freely interact with liquid within the cell. Approximately half way through the surface scan, the occurrence of the plateau-peaks again became a rarity concomitant with the return of peaks attributed to ternary complexation. During the experiment the reduced $\mathrm{MV}^{+}$. was oxidized back to its $\mathrm{MV}^{2+}$ state leading to the reappearance of the sharp peaks and a reduction in the appearance of the plateau-peak. Observation of the oxidation-dependent peaks over time confirmed our hypothesis that the plateau-peak is related to portal interactions of the $\mathrm{Np}$ moiety with the $\mathrm{CB}[8]$, not full ternary complexation. This observation is also supported by control experiments using CB[7] in place of $\mathrm{CB}[8]$, which show a rather high occurrence of plateau-peaks versus sharp peaks, further confirmation of portal interactions (see ESI, Figure S8).

Other binders and controls To examine the widespread application of this SMFS method for understanding interactions between molecules in a ternary complex, or to answer the question 'do these molecules form a ternary complex in $\mathrm{CB}[8]$ ?', $\mathrm{PDMA}_{12 k}$ was functionalized with a methoxy phenyl end group, denoted Pm-DMA $12 k$. The $\mathrm{K}_{a}$ value for the methoxy phenyl moiety with $\mathrm{MV}^{2+} \cdot \mathrm{CB}[8]$ is approximately two orders of magnitude lower than that of naphthol. ${ }^{30}$ Thus, it should be possible to observe a reduced interaction force for the 
methoxy phenyl end group compared to Np-DMA $12 k$ by SMFS. The results of this experiment are shown in Figure 5.

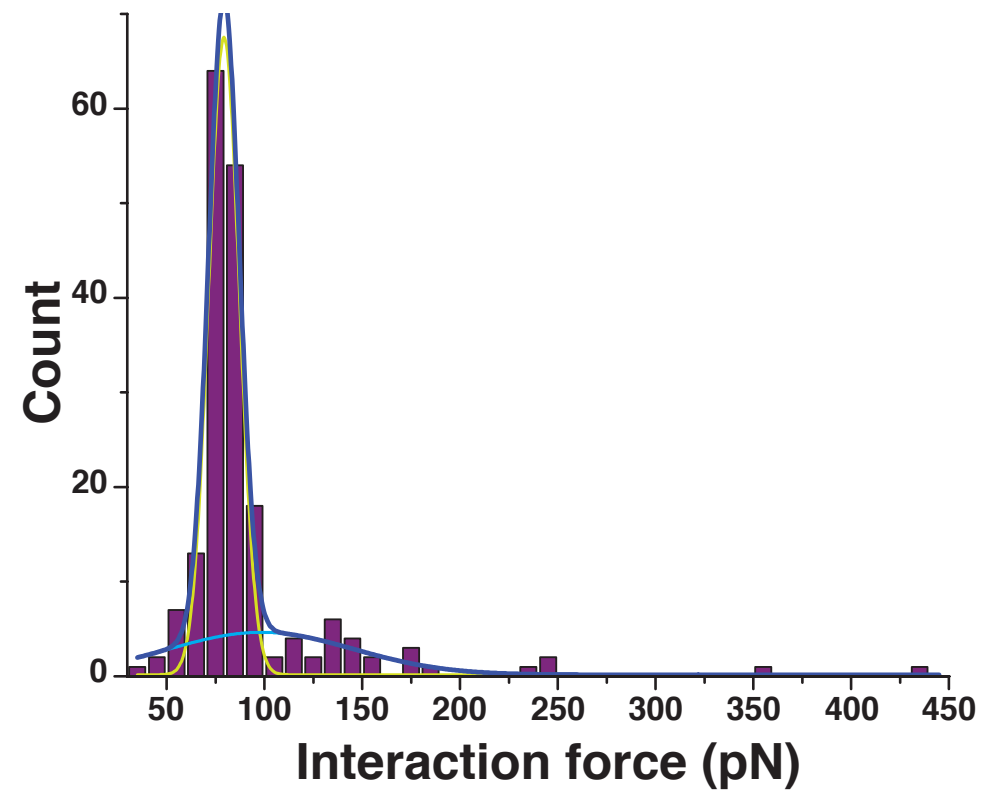

Figure 5: Histogram showing the distribution of interaction forces for Pm-DMA $12 k$

The weaker binder led to a reduction in peak heights complicating differentiation between plateau and peaks, as a result, the bimodal distribution seen previously is almost non-existent (Figure 5). The peaks are approximately $90 \mathrm{pN}$, only slightly higher an interaction force than the plateau, which remains in the region of $80 \mathrm{pN}$. It is therefore possible to determine the difference between second guests in a heteroternary $\mathrm{CB}[8]$ complex using SMFS. Furthermore, the changes observed in the interaction forces correlates with the changes in association constant of these second guests. More investigation is required to determine if a direct correlation between interaction force and association constant can indeed be determined from this method. 


\section{Conclusion}

Understanding complex formation on the single-molecule scale is key to discerning the link between molecular and macroscale behavior of supramolecular systems. Dynamic systems such as CB[8] ternary complexes have immense applicability in biomedicine, sensors, adhesives, drug delivery, and bioelectronics, to name a few but the link between behavior at different length scales needs to be more fully understood before control and manipulation of the systems can be exploited in myriad applications. This work has led to the quantification of the interaction force between methyl viologen $\cdot \mathrm{CB}[8]$ and naphthol, yielding a ternary complex. Moreover, it was possible to observe two additional interaction forces, hydrophobic and portal interactions, the interaction forces of which were also quantified. We also gained a better understanding of the role of spacers and counterions in the potential interactions of the ternary complex, providing a greater understanding of the role of counterions in adhesive behavior. Such information brings us closer to a full understanding of complex formation in host-guest supramolecular systems on a variety of length scales.

\section{Author Information}

Corresponding Author *Z. Walsh-Korb. E-mail: walshkorb@unistra.fr and *O.A. Scherman. E-mail: oas23@cam.ac.uk

Note The authors declare no competing financial interest.

\section{Acknowledgement}

The authors thank Y. Wu for help with the functionalization of the mica surfaces and C.S.Y. Tan for providing the Np-PEG 5 for the ITC experiments performed in this work. This work was supported in part by the Engineering and Physical Sciences Research Council (EPSRC), 
the Walters-Kundert Charitable Trust and an ERC Starting Investigator grant (ASPiRe, 240629). ZWK, ERJ, YL thank the Royal Society of Chemistry for a grant allowing travel to Tsinghua University to carry out this research. YY would like to acknowledge financial support from the Young Scientists of the National Science Foundation of China (21304052). YL thanks the Chinese Overseas Scholarship Trust for financial support. JdB thanks the Marie Curie Actions program for financial support. PEW thanks the Atomic Weapons and Energy Commission and the Melville Laboratory for Polymer Synthesis for financial support.

\section{Supporting Information Available}

Detailed experimental procedures and characterization data are available in the electronic supplementary information

This material is available free of charge via the Internet at http://pubs.acs.org/.

\section{References}

(1) Yamaguchi, H.; Kobayashi, Y.; Kobayashi, R.; Takashima, Y.; Hashidzume, A.; Harada, A. Photoswitchable Gel Assembly Based on Molecular Recognition. Nat. Commun. 2012, 3, 603 .

(2) Ahn, Y.; Jang, Y.; Selvapalam, N.; Yun, G.; Kim, K. Supramolecular Velcro for Reversible Underwater Adhesion. Angew. Chem. Int. Ed. 2013, 52, 3140-3144.

(3) Blass, J.; Bozna, B. L.; Albrecht, M.; Krings, J. A; Ravoo, B. J.; Wenz, G.; Bennewitz, R. Switching Adhesion and Friction by Light Using Photosensitive Guest-Host Interactions. Chem. Commun. 2015, 51, 1830-1833.

(4) Nakamura, T.; Takashima, Y.; Hashidzume, A.; Yamaguchi, H.; Harada, A. A Metal- 
Ion-Responsive Adhesive Materials via Switching of Molecular Recognition Properties. Nat. Commun. 2014, 5, 1-9.

(5) Ruffatto III, D.; Parness, A.; Spenko, M. Improving Controllable Adhesion on Both Rough and Smooth Surfaces with a Hybrid Electrostatic/Gecko-like Adhesive. J. $R$. Soc. Interface 2014, 11, 20131089.

(6) Lee, H.; Scherer, N. F.; Messersmith, P. B. Single-Molecule Mechanics of Mussel Adhesion. Proc. Nat. Acad. Sci. USA 2006, 103, 12999-13003.

(7) Autumn, K.; Peattie, A. Mechanisms of Adhesion in Geckos. Integr. Comp. Biol. 2002, 42, 1081-1090.

(8) Kim, J.; Jung, I.; Kim, S.; Lee, E.; Sakamoto, S.; Yamaguchi, K.; Kim, K. New Cucurbituril Homologues: Syntheses, Isolation, Characterization and X-ray Crystal Structures of Cucurbit[n] uril (n=5,7 and 8). J. Am. Chem. Soc. 2000, 122, 540-541.

(9) An, Q.; Brinkmann, J.; Huskens, J.; Krabbenborg, S.; de Boer, J.; Jonkheijm, P. A Supramolecular System for the Electrochemically Controlled Release of Cells. Angew. Chem. Int. Ed. 2012, 51, 12233-12237.

(10) Kang, J. K.; Hwang, I.; Ko, Y. H.; Jeon, W. S.; Kim, H. J.; Kim, K. Electrochemically Controllable Reversible Formation of Cucurbit[8]uril-Stabilzed Charge-Transfer Complex on Surface. Supramol. Chem. 2008, 20, 149-155.

(11) Tian, F.; Cheng, N.; Nouvel, N.; Geng, J.; Scherman, O. A. Site-Selective Immobilization of Colloids on Au Substrates via a Noncovalent Supramolecular 'Handcuff'. Langmuir 2010, 26, 5323-5328.

(12) Gonzalez-Campo, A.; Brasch, M.; Uhlenheuer, D. A.; Gomez-Casado, A.; Yang, L. T.; Brunsveld, L.; Huskens, J.; Jonkheijm, P. Supramolecularly Orientated Immobilization of Proteins Using Cucurbit[8]uril. Langmuir 2012, 28, 16364-16371. 
(13) Gonzalez-Campo, A.; Eker, B.; Gardiniers, H. J. G. E.; Huskens, J.; Jonkheijm, P. A Supramolecular Approach to Enzyme Immobilization in Micro-channels. Small 2012, 8, 3531-3537.

(14) Harada, A.; Kobayashi, R.; Y. Takashima, Y.; Hashidzume, A.; Yamaguchi, H. Macroscopic Self-Assembly Through Molecular Recognition. Nat. Chem. 2011, 3, 34-37.

(15) Hashidzume, A.; Zheng, Y.; Takashima, Y.; Yamaguchi, H.; Harada, A. Macroscopic Self-Assembly Based on Molecular Recognition: Effect of Linkage between Aromatics and the Polyacrylamide Gel Scaffold, Amide versus Ester. Macromolecules 2013, 46, 1939-1947.

(16) Kakuta, T.; Takashima, Y.; Nakahata, M.; Otsubo, M.; Yamaguchi, H.; Harada, A. Preorganized Hydrogel: Self-Healing Properties of Supramolecular Hydrogels Formed by Polymerization of HostGuest-Monomers that Contain Cyclodextrins and Hydrophobic Guest Groups. Adv. Mater. 2013, 25, 2849-2853.

(17) Kobayashi, Y.; Takashima, Y.; Hashidzume, A.; Yamaguchi, H.; Harada, A. Reversible Self-Assembly of Gels Through Metal-Ligand Interactions. Sci. Rep. 2013, 3, 1243.

(18) Kretschmann, O.; Choi, S. W.; Miyauchi, M.; Tomatsu, I.; Harada, A.; Ritter, H. Switchable hydrogels obtained by supramolecular cross-linking of adamantyl-containing LCST copolymers with cyclodextrin dimers. Angew. Chem. Int. Ed. 2006, 45, 43614365 .

(19) Nakahata, M.; Takashima, Y.; Yamaguchi, H.; Harada, A. Redox-Responsive SelfHealing Materials Formed from Host-Guest Polymers. Nat. Commun. 2011, 2, 511.

(20) Neirynck, P.; Brinkmann, J.; An, Q.; van der Schaft, D. W. J.; Milroy, L. G.; Jonkheijm, P.; Brunsveld, L. Supramolecular Control of Cell Adhesion via FerroceneCucurbit[7]uril Host-Guest Binding on Gold Surfaces. Chem. Commun. 2013, 49, 36793681. 
(21) Gomez-Casado, A.; Jonkheijm, P.; Huskens, J. Recognition Properties of Cucurbit[7]uril Self-Assembled Monolayers Studied with Force Spectroscopy. Langmuir 2011, 27, $11508-11513$.

(22) Gomez-Casado, A.; Dam, H. H.; Yilmaz, M. D.; Florea, D.; Jonkheijm, P.; Huskens, J. Probing Multivalent Interactions in a Synthetic Host-Guest Complex by Dynamic Force Spectroscopy. J. Am. Chem. Soc. 2011, 133, 10849-10857.

(23) Kim, J.; Kim, Y.; Baek, K.; Ko, Y. H.; Kim, D.; Kim, K. Direct Force Measurement Between Cucrbit[6]uril and Spermine Using Atomic Force Microscopy. Tetrahedron 2008, $64,8389-8393$.

(24) Hu, C.; Lan, Y.; Tian, F.; West, K. R.; Scherman, O. A. Facile Method for Preparing Surface-Mounted Cucurbit[8]uril-Based Rotaxanes. Langmuir 2014, 30, 10926-10932.

(25) Sankaran, S.; van Weerd, J.; Voskuhl, J.; Karperien, M.; Jonkheijm, P. Photoresponsive Cucurbit[8]uril-Mediated Adhesion of Bacteria on Supported Lipid Bilayers. Small 2015, 11, 6187-6196.

(26) Zhang, Y.; Yu, Y.; Jiang, Z.; Xu, H.; Wang, Z.; Zhang, X.; Oda, M.; Ishizuka, T.; Jiang, D.; Chi, L.; Fuchs, H. Single-Molecule Study on the Intermolecular Interaction between C60 and Porphyrin Derivatives: Toward Understanding the Strength of Multivalency. Langmuir 2009, 25, 6627-6632.

(27) Cui, S.; Pang, X.; Zhang, S.; Yu, Y.; Ma, H.; Zhang, X. Unexpected TemperatureDependent Single Chain Mechanics of Poly(N-Isopropyl-Acrylamide) in Water. Langmuir 2012, 28, 5151-5157.

(28) Tan, X.; Litau, S.; Zhang, X.; Müller, J. Single-Molecule Force Spectroscopy of an Artificial DNA Duplex Comprising a Silver(I)-Mediated Base Pair. Langmuir 2015, 31, 11305-11310. 
(29) Biedermann, F.; Appel, E. A.; del Barrio, J.; Gruendling, T.; Barner-Kowollik, C.; Scherman, O. A. Postpolymerization Modification of Hydroxyl-Functionalized Polymers with Isocyanates. Macromolecules 2011, 44, 4828-4835.

(30) Rauwald, U.; Biedermann, F.; Deroo, S.; Robinson, C. V.; Scherman, O. A. Correlating Solution Binding and ESI-MS Stabilities by Incorporating Solvation Effects in a Confined Cucurbit[8]uril System. J. Phys. Chem. B 2010, 114, 8606-8615.

(31) Hinterdorfer, P.; Dufrêne, Y. F. Detection and Localization of Single Molecular Recognition Events Using Atomic Force Microscopy. Nat. Meth. 2006, 3, 347-355.

(32) Ren, X.; Wu, Y.; Clarke, D. E.; Liu, J.; Wu, G.; Scherman, O. A. Surface-Bound Cucurbit[8]uril Catenanes on Magnetic Nanoparticles Exhibiting Molecular Recognition. Chem. Asian. J. 2016, 10.1002/asia.201600875.

(33) Rauwald, U.; Scherman, O. A. Supramolecular Block Copolymers with Cucurbit[8]uril in Water. Angew. Chem. Int. Ed. 2008, 47, 3950-3953.

(34) Cheng, H.-B.; Zhang, Y.-M.; Xu, C.; Liu, Y. Photoresponsive Supramolecular Complexes as Efficient DNA Regulator. Sci. Rep. 2014, 4, 4210.

(35) Ratto, T. V.; Langry, K. C.; Rudd, R. E.; Balhorn, R. L.; Allen, M. J.; McElfresh, M. W. Force Spectroscopy of the Double-Tethered Concanavalin A Mannose Bond. Biophys. J. 2004, 86, 2430-2437.

(36) Ray, C.; Brown, J. R.; Akhremitchev, B. B. Correction of Systematic Errors in SingleMolecule Force Spectroscopy with Polymeric Tethers by Atomic Force Spectroscopy. J. Phys. Chem. B 2007, 111, 1963-1974.

(37) Ros, R.; Schwesinger, F.; Anselmetti, D.; Kubon, M.; Schäfer, R.; Plückthun, A.; Tiefenauer, L. Antigen Binding Forces of Individually Addressed Single-Chain Fv Antibody Molecules. Proc. Nat. Acad. Sci. USA 1998, 95, 7402-7405. 
(38) Schumakovitch, I.; Grange, W.; Strunz, T.; Bertoncini, P.; Güntherodt, H. J.; Hegner, M. Temperature Dependence of Unbinding Forces between Complementary DNA Strands. Biophys. J. 2002, 82, 517-521.

(39) Schwesinger, F.; Ros, R.; Strunz, T.; Anselmetti, D.; Güntherodt, H. J.; Honegger, A.; Jermutus, L.; Tiefenauer, L.; Plückthun, A. Unbinding Forces of Single AntibodyAntigen Complexes Correlate with their Thermal Dissociation Rates. Proc. Nat. Acad. Sci. USA 2000, 97, 9972-9977.

(40) Riener, C. K.; Stroh, C. M.; Ebner, A.; Klampl, C. W.; Gall, A. A.; Romanin, C.; Lyubchenko, Y. L.; Hinterdorfer, P.; Gruber, H. J. Simple Test System for Single Molecule Recognition Force Microscopy. Anal. Chim. Acta. 2003, 479, 59-75.

(41) Hu, C.; Lan, Y.; West, K. R.; Scherman, O. A. Cucurbit[8]uril-Regulated Nanopatterning of Binary Polymer Brushes via Colloidal Templating. Adv. Mater. 2015, 27, $7957-7962$. 


\section{Graphical TOC Entry}

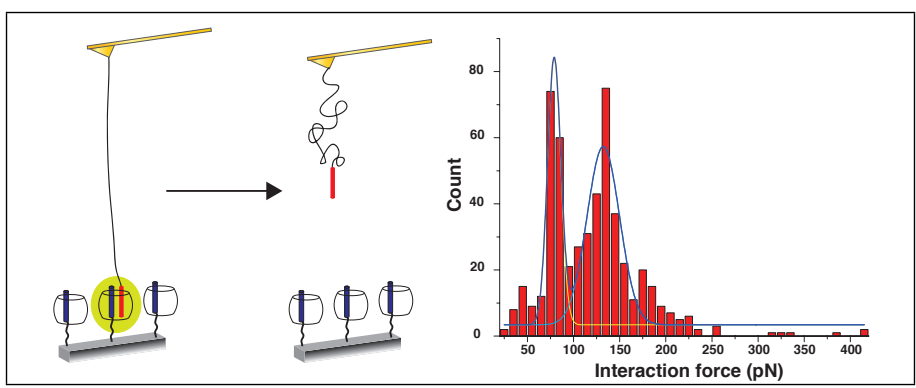

This paper describes the use of single molecule force spectroscopy to estimate the binding interactions of cucurbit[8]uril ternary complexes on surfaces for further application in adhesive materials. 